\title{
EVALUATION OF DIFFERENT KINDS OF OCCLUSAL SPLINTS THERAPY IN THE MANAGEMENT OF MYOFASCIAL PAIN
}

\author{
Mahmoud El-Moutassim Bellah El-Homossany* and Hebatallah Tarek Abdallah*
}

\begin{abstract}
The aim of the study was to compare patient satisfaction with different types of stents using visual analogue scale and Pain report analysis.

Materials and methods: Thirty dentulous patients were selected, based on the clinical examination, the patients were suffering from pain in the temporomandibular joint region that might be worse in the morning or associated with eating,tenderness of temporomandibular joint with palpation at rest and/or at function, pain and/or tenderness of masseter and temporalis muscle. The patients were randomly divided into three equal groups, ten patients in each group. Splints were constructed for the upper arch of each patient. Soft, hard and 3 Dprinted stents were fabricated. Pain intensity questionnaire (visual analogue scale) and Pain report analysis were used before treatment, after three months and six months.
\end{abstract}

Results: Kruskal-Wallis test was used to analyze the ordinal scale date of the visual analogue scale and pain report analysis. There was significance difference between the three groups. (Soft, hard and three D printed) after three month and six month of using the stent. Wicoxon test was used to test for significance between the different groups at three months and six months. The Wilcoxon test for visual analogue scale at three months showed that the hard and 3D printed stent are statistically significant to soft splint. The same was for the six months period. While Wilcoxon test for pain report analysis at 3 months didn't show any statistical significance between the three groups, while at six month there was statistical significance between the hard splint and the $3 \mathrm{D}$ printed splint with the soft splint. Friedman's test was used to compare between different time intervals inside the groups. There was a statistical significance between the three groups during the three months and six months interval time of the treatment.

Conclusion: All patients improved over time. The results showed that both hard and soft splints are effective but the hard splint and the three D printed splints are more effective compared to soft splint.

* Lecturer Removable Prosthodontics Department, Faculty of Dentistry, Ain Shams University. 


\section{INTRODUCTION}

Tempromandibular disorders (TMD) is a collective term embracing all the problems relating to tempromandibular joint (TMJ) and related musculoskeletal masticatory structures. It refers to a cluster of disorders characterized by pain in the periauricular region, pain in TMJ, or the masticatory muscles ,limitation or deviation in mandibular range of motion and noises in TMJ during mandibular function. ${ }^{(1,2)}$

The etiology of TMDs is complex and multifactorial. Numerous factors can contribute to TMDs, factors that increase the risk of TMDs are called predisposing factors which can be systemic, psychological (personality and behavior), or structural (occlusion, joint), while factors that cause the onset of TMDs are called initiating factors (trauma, overloading, or Para function habits), and factors that interfere with healing or enhance the progression of TMDs are called perpetuating factors which includes behavioral, social and emotional problems and other forms of stress and general health . In some instances a single factor may serve one or all of these roles. Thus successful management of TMDs depends on identifying and controlling these contributing factors. ${ }^{(3)}$

\section{Treatment Modalities of tempromandibular disorders}

Treatment of tempromandibular joint disorders may need one or more of the following treatment modalities:

1. Psychotherapy (emotional stress therapy); which includes patient awareness; relaxation therapy using biofeedback technique. Psychotherapy is done to tolerate noxious stimuli among individuals and from time to time in the same individual. ${ }^{(1)}$

2. Drug therapy; which includes analgesics, muscle relaxants and nutritional supplements. ${ }^{(1)}$

3. Occlusal therapy; which involves repositioning the mandible in a centric position by orthodontic means and /or occlusal equilibration to remove occlusal interferences (irreversible occlusal therapy) or by occlusal splints. ${ }^{(1)}$

4. Physiotherapy; which is used to relax the muscles and improve the circulation .physiotherapy includes thermotherapy, coolant therapy, electro galvanic stimulation (EGS) therapy, transcutanous electrical nerve stimulation (TENS), acupuncture and LASER. ${ }^{(4,5)}$

\section{Oral appliances}

Oral appliances may be classified or grouped according to their material, function, occlusal coverage, dental arch coverage, or a combination of more than one characteristic.

\section{According to the materials used in the appliance} construction: Polymers, as in hard acrylic or resilient vinyl splints, Metallic, as in metallic alloy or gold overlay, Combination of metal, polymers, and composite. ${ }^{(6)}$

According to function, oral appliances are classified as:

Pain Release Splint: Soft temporary resilient or hard acrylic oral appliances are used to relieve unwanted mechanical pressure that causes pain. Examples of pain release splints are vinyl or thermoplastic soft splints. ${ }^{(6)}$

Stabilizing Appliance (Michigan splint): it provides stabilization of the joint, redistribution of the forces at the tooth and /or joint level, relaxation of the elevator muscles and /or protection of the teeth from the effects of bruxism. It provides tissue stabilization, thus decreases pain, and promotes healing. A periodontal provisional appliance is used to stabilize mobile teeth. Bionator-like appliances for mandibular nocturnal use are examples of stabilizing appliances. A mandibular appliance with deep occlusal cuspal inclinations is another example of a mandibular stabilizing appliance. ${ }^{(6)}$ 
Anterior bite plane: It is also referred as relaxation plate, a Hawley plate, a Sved plate, and Dessner plate. It covers the palate and gains retention from clasps on the first maxillary molars. It has been suggested for treatment of para functional activity associated with unfavorable posterior tooth contact but only for short periods. The disadvantage of the use of this type of splint involves a risk of negatively affecting the occlusion by causing extrusion of the premolars and molars and intrusion of the incisors and canines, resulting in a frontal open bite. ${ }^{(7)}$

Posterior bite plane: Usually fabricated for mandibular teeth and consists of areas of hard acrylic located over the posterior teeth and connected by a cast metal lingual bar. The treatment goals of the posterior bite plane are to achieve major alternations in the vertical dimension and mandibular repositioning .Posterior bite planes are used in severe lost vertical dimension cases. The major concern surrounding this appliance is that it occludes with only part of the dental arch, therefore allows potential super eruption of the remaining teeth ${ }^{(7)}$

Repositioning Appliance: A device used to alter the mandible's spatial relation to the cranium to allow a proper biomechanical balance. The mandibular orthopedic repositioning appliance (MORA), anterior repositioning appliance (ARA), vertical decompression appliance (VDA), and bicuspid buildup are examples of the mandibular repositioning techniques. Appliances used for treatment of snoring or obstructive sleep apnea are mandibular-tongue repositioning appliances. ${ }^{(8,9)}$

Orthodontic Appliance: A device that is used to move teeth into a desirable spatial alignment. Examples are fixed brackets and wire orthodontic appliances, and fixed or removable functional orthodontic appliances that are designed to include active parts such as screws, springs, and loops. ${ }^{(6)}$

Prosthodontic Appliance: A device that replaces missing teeth, dental, or maxillofacial structures. Examples of this type of appliance are fixed or removable partial dentures. An obturator, a prosthetic appliance, replaces missing portions of maxillofacial tissues. ${ }^{(6)}$

Protective Appliance:A device that protects the teeth or oral structures. Examples are provisional appliances that are used to decrease the effects of bruxism on the teeth, muscles, and TMJ. In addition, sports or athletic appliances are considered protective ones. ${ }^{(10-12)}$

Retraining Appliance: A device that is used to maintain tissues in position to facilitate healing. An orthodontic retainer and intermaxillary fixation devices are examples of retaining appliances. ${ }^{(6)}$

Diagnostic Appliance: A device that is used in head and neck disorders to rule out or confirm a specific diagnosis. Pain release, myalgic, and mandibular repositioning appliances may be used initially for diagnostic reasons. ${ }^{(6)}$

Hydrostatic splint: a water-bearing pressureequalizing appliance sold under the commercial name "Aqualizer" This prefabricated splint does not require dental impressions or the manufacture of customized appliances. Instead, this disposable splint can be purchased through a dentist, readymade for use, and is claimed to fit the mouths of most users. In practice, however, the pads often slip from under the teeth, the appliance wanders in the mouth, and may even be expelled during sleep. These shortcomings could be readily overcome by combining the idea of a hydrostatic splint with the taste-based appliance. The pads would be attached to the sleeves, and could be connected to each other either along the contours of the appliance, as they do now, or directly. ${ }^{(6)}$

According to the amount of occlusal coverage oral appliances can be: Full occlusal coverage appliance that covers all the teeth in the dental arch as in Tanner, Ricketts, and others or partial occlusal coverage appliance, as in Gelb or Sved as they cover posterior teeth. On the other hand an upper anterior 
appliance with anterior biting plan and a nociceptive trigeminal inhibition (NTI) appliance cover only the anterior segment of the dental arch. ${ }^{(6)}$

According to the utilized dental arch oral appliances can be; Maxillary appliance, which covers the upper arch , Mandibular appliance; which covers the lower arch or combination appliance, as in appliances that utilize both arches for support and retention. Bionator-like devices, as well as snoring and sleep apnea appliances, are examples. ${ }^{(6)}$

It is important to mention that many intraoral appliances possess more than one function. For example, a mandibular orthopedic repositioning appliance (MORA) repositions, decompresses, stabilizes, and protects the tempromandibular and surrounding structures; a Gelb appliance is a repositioning, decompressing, and occasionally prosthetic device when replacing missing teeth. ${ }^{(6)}$

\section{The modes of action of intraoral appliances have been theorized as following:}

Occlusal Disengagement Theory: This theory indicates that an intraoral maxillary occlusal appliance constructed in a centric occlusal position reduces or eliminates muscle activity and stabilizes the TMJ. ${ }^{(6)}$

Vertical Dimension Restoration Theory: This theory proposes that the restoration of the vertical dimension using a properly constructed splint to regain the original vertical height between the upper and lower arches reduces the abnormal muscle activity and reduces symptoms. ${ }^{(12)}$

Maxillofacial Realignment Theory: This theory assumes that changing the unbalanced position between the mandible and maxilla to a more anatomical or neuromuscular balanced relation decreases or eliminates symptoms of TMD. ${ }^{(6)}$

Condylar Repositioning Theory: This theory conceptualizes using a repositioning splint to place the mandibular condyle in a proper position in the articulating fossa, with the disc interposing between the condyle and the posterior slope of the articulating eminence, promoting TMJ healing. In addition, this theory explains that an anterior temporary repositioning of the condyle allows recapture of the disc; a posterior repositioning of the mandible to its original position is accomplished by gradual posterior adjustment of the splint. ${ }^{(6)}$

Cognitive Awareness Theory: This theory proposes that having an occlusal appliance on the teeth constantly reminds the individual not to place undue stress on the mandible that causes harmful muscle activity. ${ }^{(7)}$

\section{Occlusal splint}

Occlusal splint therapy may be defined as the art and science of parafunctional neuromuscular harmony in the masticatory system by creating a mechanical disadvantage for parafunctional forces with removable appliances. ${ }^{(13)}$

Occlusal splint is a diagnostic, relaxing, repositioning and reversible device. According to the Glossary of Prothodontics terms $8^{\text {th }}$ edition, occlusal splint is defined as any removable artificial occlusal surface used for diagnosis or therapy affecting the relationship of the mandible to the maxilla. It may be used for occlusal stabilization, for treatment of tempromandibular disorders, or to prevent wear of the dentition. ${ }^{(7)}$

Occlusal splint has many functions it may be one or more of the following ;stabilization of the tempromandibular joint and dental occlusion, relief tempromandibular joint (TMJ) overloading, decrease of occlusal interference, Reduction of masticatory muscle activity, Alleviation of oral parafunctional activities, Protecting the teeth from further breakdown and Inducing a placebo effect. ${ }^{(7)}$

\section{Hard occlusal splint :}

Most of the occlusal splints are typically constructed of hard acrylic resin. As the resilient or soft type is less durable, more difficult to adjust and harder to keep clean. In some patients, soft 
splints may encourage clenching and grinding, and therefore, increase the symptoms. Hard occlusal splints have limitations in their use,they cannot be used in children for more than short periods because they may not fit after a relatively short time and therefore interfere with the normal growth pattern. A soft splint may also be indicated in adult patients who cannot adjust to a conventional splint because they feel it is too tight, creating tension. ${ }^{(1)}$

\section{Soft occlusal splint}

This is the most commonly prescribed occlusal splint. It is quick to fabricate and can be provided as emergency treatment for a patient who presents with an acute TMD..$^{(9)}$

This splint is more readily tolerated in the lower arch than the upper arch as there is no satisfactory way of thinning the margins of the splint while keeping good retention. This means that if the splint is made on the upper arch, the patient is subjected to a thick ridge of polyvinyl in the palate which often makes the splint difficult to tolerate. ${ }^{(14)}$

The only record needed is a lower alginate impression as the splint is not made to a specific occlusal prescription although it is cheap and easy to fabricate, it cannot be readily adjusted and It should be clear to the patient that, in approximately $10 \%$ of cases, these appliances will make the symptoms worse. This is especially true in patients who are 'dedicated bruxists' as they are so aware of having something compressible in the mouth, they actually increase the activity rather than decrease it. ${ }^{(14)}$

These appliances are usually worn only during sleep at night and, if they are to be successful, will produce some symptomatic relief within six weeks of use. They should be replaced after 4-6 months as they lose their resilience with time..$^{(14)}$

The appliance is generally made out of 2 $\mathrm{mm}$ polyvinyl. If a thinner splint is required, the technician can be instructed to overheat the material before vacuum forming, and if a selectively thicker appliance is required (for instance in a patient with an anterior open bite) then layers can be added in certain areas (i.e. anteriorly) to ensure even occlusal contact. $^{(14)}$

Soft resilient splints are used with children and adolescents with primary or mixed dentition because hard acrylic splints interfere with jaws and teeth development. Athletics also use soft splints to protect their teeth and para oral structures from traumas also patients expressing high level of clenching and bruxism use it to dissipate some heavy loads during para function habits.(14)

Resilient splints have many advantages where they can be easily fabricated at chair side in $15 \mathrm{~min}$ and used in acute situations, with complete denture wearers, in patients who have fixed partial dentures supported by osseointegrated implants and used by patients who must wear an appliance during day as its placement in mandible makes it less visible, but one drawback of the resilient appliances is that they deteriorate fairly quickly, thus can be used for only a short period, after which new one must be made if the patient still needs to use it. ${ }^{(9)}$

\section{MATERIALS AND METHODS}

Thirty dentulous patients were selected from the outpatient clinic of diagnosis department and prosthodontic department, Faculty of dentistry, Ain shams university.

Patients were selected with range of age from 18 to $60 \mathrm{y}$, no sex predilection, suffer from bruxism from 2 months up to 2 years.

\section{Inclusion criteria:}

Based on the clinical examination , the patients were suffering from some or all of the following signs and symptoms, and were considered to suffer from one of the parafunctional habits "Bruxism"

1. Pain in the temporomandibular joint region that might be worse in the morning or associated with eating. 
2. Tenderness of temporomandibular joint with palpation at rest and/or at function.

3. Pain and/or tenderness of masseter and temporalis muscle.

4. Intra orally on examining the teeth for occlusion, mobility and any special finding for bruxism as wear of teeth presenting as multiple ,smooth and shiny facets.

5. The patient should have trigger points.

\section{Exclusion criteria:}

1. Pregnancy or breast feeding.

2. Prior Botox treatment.

3. Trigeminal neuralgia.

4. Pain of dental origin

5. Muscle relaxant use.

6. Aminoglycoside use.

7. Rheumotoid arthritis.

8. Neuropathic pain.

9. Vulnerable groups (prisoners, mental disorders, patients not capable of decision making).

10. Any local skin infections over the masseter or temporalis muscle.

11. Patients with low vertical dimension.

12. Patients were having TMJ sounds.

13. Smokers

\section{2- Diagnosis}

For proper diagnosis case history was taken, and clinical examination was performed.

\section{A-History:}

Case history of all patients was recorded including personal, dental,medical, and sleep history. Using a two printed questionnaire; one to collect data about bruxism and the other to collect data about pain intensity using visual analogue scale.

\section{1- Personal and dental history:}

Personal, and dental history were assessed using the following bruxism questionnaire:

\begin{tabular}{|l} 
Date \\
1. Name \\
Age \\
Sex \\
Occupation \\
Address \\
\hline 2. Which of the following do you have (circle all \\
that apply) \\
Headache Neck pain Jaw pain \\
Ear pain Facial pain Other \\
\hline
\end{tabular}

3. Which side hurts (circle one)

Right Left Both

- How long have you had this pain?

- Is the pain constant?

- Is the pain (circle all that apply) Aching Burning Stabbing Other

- Is the pain the worst in the (circle all that apply) Morning Afternoon

- Evening Night

-What makes the pain better?

- What makes the pain worse?

-What medication(s) do you take or have you previously taken for your pain?

Medications

Dose

Frequency

\begin{tabular}{|lccc|}
\hline 4. Does it hurt to chew? & Y & $\mathrm{N}$ \\
\hline 5. Does it hurt to open wide? & $\mathrm{Y}$ & $\mathrm{N}$ \\
\hline $\begin{array}{l}\text { 6. Which side of your jaw makes a popping noise? } \\
\text {-Which side of your jaw makes a clicking noise? }\end{array}$ & $\mathrm{R}$ & $\mathrm{L}$ & $\mathrm{L}$ \\
\hline 7. Has your jaw ever locked? & $\mathrm{Y}$ & $\mathrm{N}$ \\
-When did this first happen? & & \\
-When did this last happen? & & \\
\hline 8. Has your jaw ever slipped out of place? & $\mathrm{Y}$ & $\mathrm{N}$ \\
- Which side? & $\mathrm{R}$ & $\mathrm{L}$ \\
\hline
\end{tabular}




\begin{tabular}{|lr|}
\hline 9. Have you noticed a change in you bite? & Y N \\
\hline 11..Do you have tongue indentations & $\mathrm{Y} \mathrm{N}$ \\
\hline 12. Do you have problems with your ears? & $\mathrm{Y} \mathrm{N}$ \\
-Dizziness? & $\mathrm{Y} \mathrm{N}$ \\
-Ringing? & $\mathrm{Y} \mathrm{N}$ \\
-Hearing? & $\mathrm{Y} \mathrm{N}$ \\
-Other? & $\mathrm{Y} \mathrm{N}$ \\
\hline 13. Is it difficult to swallow? & $\mathrm{Y} \mathrm{N}$ \\
-Is it painful to swallow? & $\mathrm{Y} \mathrm{N}$ \\
-Have you noticed lumps in your face? & $\mathrm{Y} \mathrm{N}$ \\
-Throat? & $\mathrm{Y} \mathrm{N}$ \\
-Neck? & $\mathrm{Y} \mathrm{N}$ \\
-Other? & \\
\hline 14. Have you had any prior treatment for Bruxism? & $\mathrm{Y} \mathrm{N}$ \\
-Splint? Y N When? Did it help? & $\mathrm{Y} \mathrm{N}$ \\
-Bite Adjustment ? Y N When? Did it help? & $\mathrm{Y} \mathrm{N}$ \\
-Orthodontics? Y N When? Did it help? & $\mathrm{Y} \mathrm{N}$ \\
-Other? & \\
\hline
\end{tabular}

\section{2- Medical history includes:}

Patient having any of the following should be excluded:

1. Any systemic disease affecting bone or muscle including rheumatoid arthritis .

2. Ear infection.

3. Periocular pain.

4. Any medications especially steroids, and muscle relaxant.

\section{3- Sleep history:}

History of any sleep disorder and type of treatment the patient follows if present. Transient sleep interruptions should be taken into consideration.

\section{4- Pain intensity questionnaire (visual analogue scale):}

The Visual Analogue Scale (VAS) is a singleitem measure. It was performed before treatment ,after 3 months and 6 months to monitor patients satisfaction and improvement of pain with each treatment modality .
A VAS most commonly consists of a $100 \mathrm{~mm}$ horizontal line anchored with two opposite labels; patients mark a score on the scale using a vertical line. The VAS for pain consisted of four $100 \mathrm{~mm}$ lines, each labeled at the left end as no pain $(0 \mathrm{~mm})$ and at the right end as 'very severe pain' $(100 \mathrm{~mm})$. Patients were asked to draw a vertical mark on each line, one on the upper line for their current pain before starting treatment, one on the second line for the pain intensity after first follow up,one on the third line for the pain intensity after second follow fig.(1).

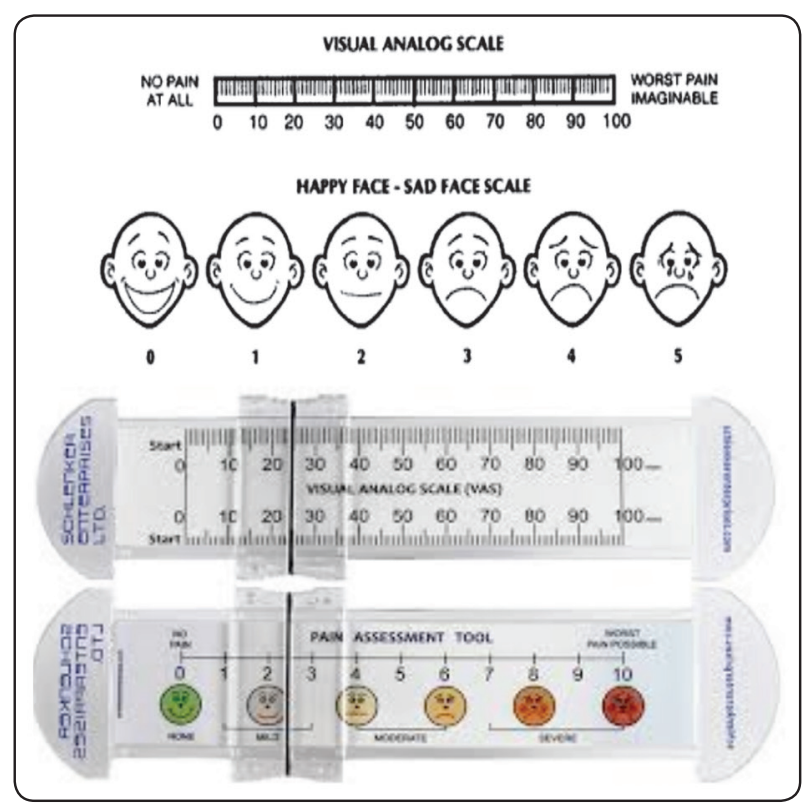

Fig. (1) Visual analog scale (0-10 VAS)and it was categorized using the following terms : no pain (0), mild (1-3), moderate (4-6), severe (7-9), or worse pain (10).

\section{B- Clinical examination}

Patients clinical examination was carried out including TMJ, muscles of mastication and intraoral examination.

\section{1- Temporomandibular joint (TMJ) examination:}

\section{a- Inspection:}

Inspection of the skin covering the TMJ region for any swelling, exudates, bruises, skin infection, scar or any other abnormalities. 


\section{$b$-Tenderness:}

Bilateral palpation of the condyles during both rest and function.

\section{c-Joint sounds:}

Joints sounds are assessed by palpation during opening and closing, thus patients with joint sounds were excluded from this study.

\section{2- Muscles of mastication examination:}

\section{a-Masseter muscle}

The deep part of the masseter muscle was palpated extra orally just below the zygomatic arch and approximately $10 \mathrm{~mm}$ in front of the condylar head, while the anterior part of the superficial muscle was palpated bidigitally from the origin in the zygomatic arch to the insertion at the angle of the mandible.

\section{b- Temporalis muscle}

Temporalis muscle was palpated at its origin on the temporal fossa on the lateral surface of the skull and its insertion was palpated intra-orally at the retromolar fossa with the index.

\section{3- Intra oral examination}

Examination for malocclusion, attrition, mobility and any special findings for bruxism as wear of tooth presenting as multiple,smooth and shinny facets at cusp tip and incisal edges with brown discoloration of exposed dentin and / or broken filling or tooth.

\section{Grouping of the patients}

The patients were randomly divided into 3 equal groups, ten patients in each group. Splints were constructed for the upper arch of each patient.
The fabrication of the stent was made as follow:

- An impression of both maxillary and mandibular arches was made using alginate* in a proper sized stock tray. Then poured immediately using stone.

- The maxillary cast was mounted on the semiadjustable articulator ${ }^{* *}$ by means of a face bow** record and centric occlusion record was used to mount the mandibular cast.

- Vertical opening of the articulator was done, to gain $2 \mathrm{~mm}$ space between the posterior teeth, representing the thickness of the future splint.

- A protrusive record was done and both the protrusive and lateral condylar guidance were adjusted on the articulator.

Group A: For the soft splint, a vacuum pressure molding device was used for fabrication with 2-mm-thick of resilient vinyl sheets measuring 13 $\mathrm{cm}$. The rubber sheet was completely and properlyadapted to the cast in the vacuum former. The sheet wasremoved, and sharp scissors were used to trim the splint edges. The palatal portion of the splint was removed to obtain theshape.fig(2)

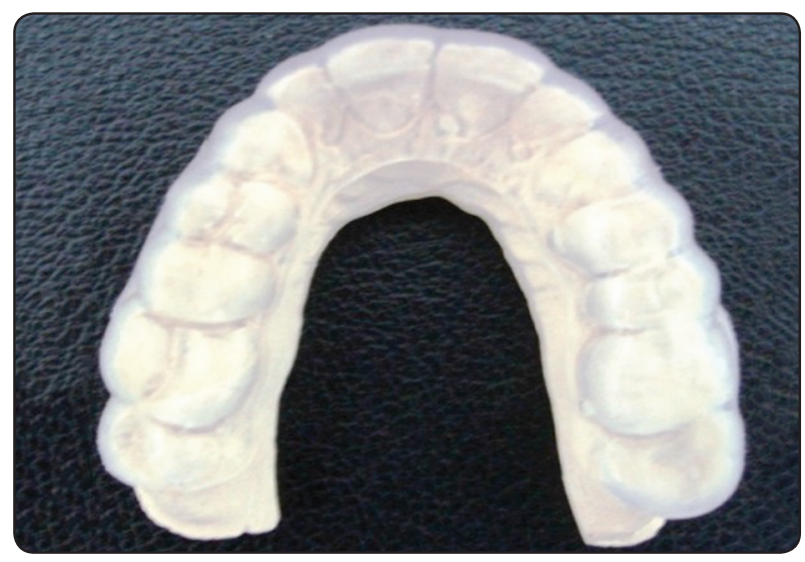

Fig. (2) Soft splint

\footnotetext{
* Cavex alginate, Cavex, Holland

** Dentatus, Dentatus AB, Sweden.
} 
Group B: For the hard splint, self-curing transparent acrylic resin was used to fabricate the splint in the form of a flat anterior bite plane with a thickness of 2-3 mm, which separated the posterior teeth while allowingcontact between the anterior teeth. fig(3)

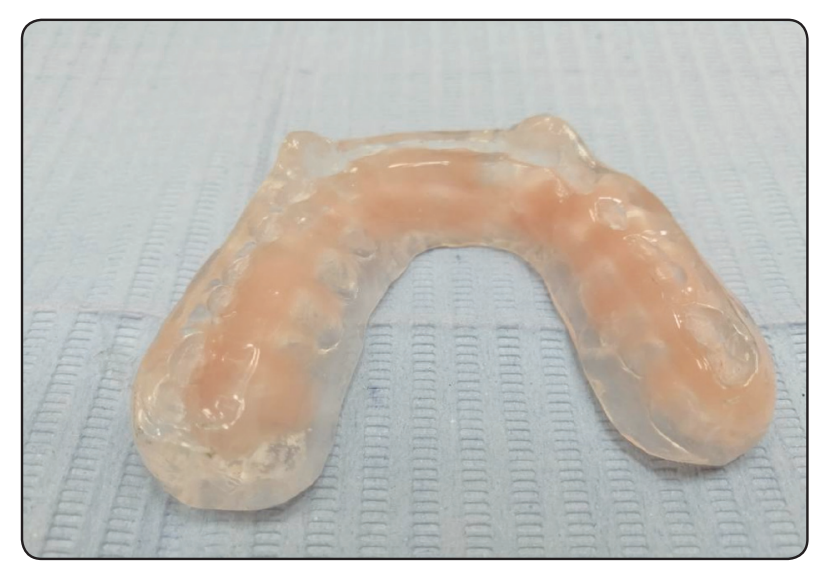

Fig. (3) Hard splint

Group C: Patients in this group received hard occlusal stabilization splint constructed on the maxillary teeth of $2 \mathrm{~mm}$ thickness by 3Ddigital printing. fig $(4,5)$

- An intraoral scanner* was used to scan both casts on the articulator mounted at the desired thickness of the splint, then both protrusive and lateral values (bennet angle) taken from the articulator was introduced to the software ${ }^{* *}$ on the virtual articulator

- A bar was added to the splint shell virtual cast, where the occlusal surface was adjusted to be flat, and achieve contact with the working cusps of all the mandibular premolars and molars.

- Ramps created at the canine region, to create disocclusion of the posterior teeth during lateral and protrusive movement.
- The virtual articulator simulate the protrusive and lateral movement based on the date introduced for lateral and protrusive movements, to check that ramps cause un interrupted disocclusion of posterior teeth represented by the continuous $\mathrm{v}$ shape on the ramp .

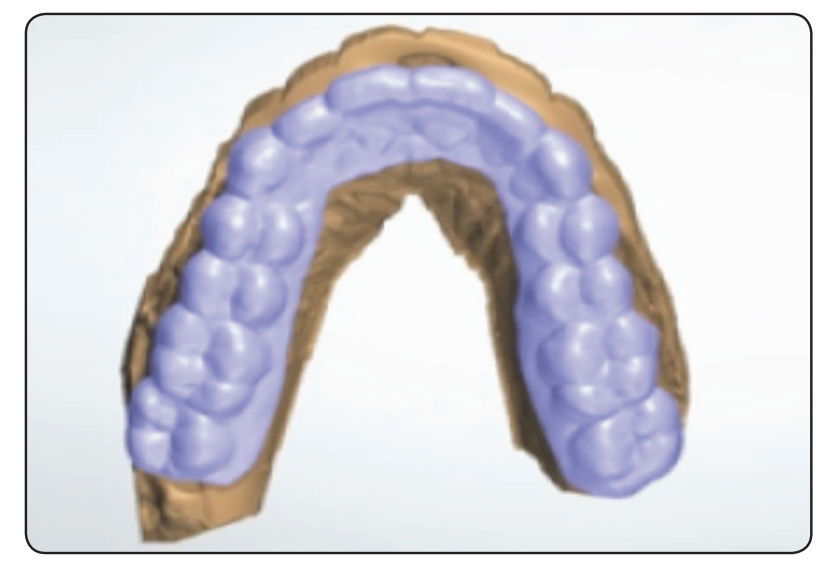

Fig. (4) Shell constructed virtually to the desired extensions on the virtual maxillary cast.

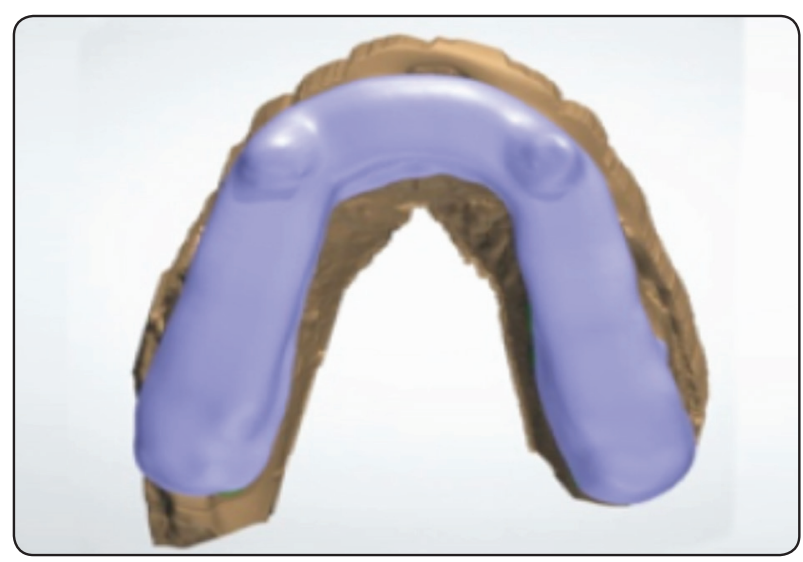

Fig. (5) Flat bar with ramps in the canine region constructed over the shell to achieve a flat surface to contact only with the buccal cusps of the lower teeth.

- The splint was printed using 3D printer ${ }^{* * *}$ and the splint was checked in the patient mouth for retention, and proper disocclusion of the posterior teeth during lateral and protrusive

* Planmeca plan scan,650 International Parkway, Richardson, Texas 75081, USA.

** 3Shape CAD,3Shape A/S • HolmensKanal 7, 4. 1060 Copenhagen K Denmark

*** Perfactory Desktop Digital Dental 3D Printer (DDDP), envisionTec., 
movements using an articulating paper placed at the area of the canine ramps, and asking the patient to protrude the mandible and move from one side to other side, interrupted v-shape was produced.

The patient gradually weaned off the splint after 6 months, but told to wear it if their discomfort returns which is often at times of stress.

Records were performed for each patient in the three groups at the following timings:

- Preoperative record, before starting the treatment.

- Post operative , 3 months after beginning of the treatment.

- After 6 months of the beginning of the treatment.

- Subjective evaluation: Pain intensity was monitored using the VAS during the previously mentioned follow up periods

- Objective evaluation: Pain report analysis Muscular palpation (masseter, temporalis and pterygoid muscles) was performed bilaterally with tight and constant pressure and were classified on a scale from 0 to 3 ( 0 , no pain; 1 , verbally reported pain; 2, pain or discomfort followed by fascial musculature contraction and 3 , when the patient backed away or showed lacrimation).

The data was collected and tabulated for statistical analysis. Kruskal-Wallis test was used to analyze the ordinal scale data of the visual analogue scale and pain report analysis. Wicoxon test was used to test for significance between the different groups at three months and six months. Friedman's test was used to compare between different time intervals inside the groups. The significance level was set at $\mathrm{P} \leq 0.05$. Statistical analysis was performed with
IBM $^{* \circledast}$ SPSS $^{* * \circledast}$ Statistics Version 20 for Windows.

\section{RESULTS}

All the patients in the three groups attended the follow up periods, then the obtained data were statistically analyzed, and graphically illustrated.

\section{Visual analogue scale}

Kruskal-Wallis test was used to analyze the ordinal scale data of the visual analogue scale. The mean of the soft splint at the beginning was 9.1 and at three months was 8.5 and at 6 months was 7.3 . The mean of the hard splint at the beginning was 8.7 and at three months was 3.1 and at 6 months was 1.98 . The mean of the $3 \mathrm{D}$ printed splint at the beginning was 8.33 and at three months was 2.09 and at 6 months was 1.67

There was significance difference between the three groups. (Soft, hard and 3D printed) after three months and six month of using the stent. The $\mathrm{P}$ value was $<0.05$.

Wilcoxon test was used to test for significance between the different groups at three months and six months. The Wilcoxon test for visual analogue scale at three months showed that the hard and 3D printed stent are statistically significant to soft splint. The $\mathrm{P}$ value was $<0.05$. The same was for the six months period. There was no statistical significance between the $3 \mathrm{D}$ printed and the hard splint in the 6 months interval $\mathrm{P}$ value $>0.05$. Friedman's test was used to compare between different time intervals inside the groups. There was a statistical significance between the three groups during the three months and six months interval time of the treatment.The $\mathrm{P}$ value was $<0.05$. 


\section{Comparison between VAS results for the three groups during the follow up periods:}

TABLE (1) Comparison between VAS results for the three groups during the follow up periods

\begin{tabular}{|l|c|c|c|c|c|c|c|}
\hline \multirow{2}{*}{} & \multicolumn{2}{|c|}{ Preoperative } & \multicolumn{2}{c|}{ After 3 months } & \multicolumn{2}{c|}{ After 6 months } & \multirow{2}{*}{ p.value } \\
\cline { 2 - 8 } & Mean & Std. & Mean & Std. & Mean & Std. & \\
\hline Soft splint & 9.1 & 0.85 & 8.5 & 0.51 & 7.3 & 057 & $\mathrm{P}<0.001$ \\
\hline Hard splint & 8.7 & 0.98 & 3.1 & 0.25 & 1.98 & 0.06 & $\mathrm{P}<0.001$ \\
\hline 3D digital splint & 8.33 & 0.81 & 2.09 & 0.26 & 1.67 & 0.58 & $\mathrm{P}<0.001$ \\
\hline P value & \multicolumn{3}{|c|}{0.1} & \multicolumn{2}{c|}{$\mathrm{P}<0.001$} & \multicolumn{3}{c|}{$\mathrm{P}<0.001$} & \\
\hline
\end{tabular}

\section{Pain report scale}

The mean of the soft splint at the beginning was 2.47 and at three months was 1.28 and at 6 months was 1.09. The mean of the hard splint at the beginning was 2.39 and at three months was 1.21 and at 6 months was 1.06 . The mean of the $3 \mathrm{D}$ printed splint at the beginning was 2.48 and at three months was 1.14 and at 6 months was 1 .

Kruskal-Wallis test was used to analyze the ordinal scale date of the Pain report scale. There was significance difference between the three groups. (Soft, hard and three D printed) after three months and six months of using the stent. The $\mathrm{P}$ value was $<0.05$. While Wilcoxon test for pain report analysis at 3 months didn't show any statistical significance between the three groups, while at six month there was statistical significance between the hard splint and the $3 \mathrm{D}$ printed splint with the soft splint. There was no statistical significance between the three D printed and the hard splint. Friedman's test was used to compare between different time intervals inside the groups. There was a statistical significance between the three groups during the three months and six months interval time of the treatment The $\mathrm{P}$ value was $<0.05$.

TABLE (2) Comparison between pain report scale results for the three groups during the follow up periods

\begin{tabular}{|l|c|c|c|c|c|c|c|}
\hline & \multicolumn{2}{|c|}{ Preoperative } & \multicolumn{2}{c|}{ After 3 months } & \multicolumn{2}{c|}{ After 6 months } & \multirow{2}{*}{ P value } \\
\cline { 2 - 8 } & Mean & Std. & Mean & Std. & Mean & Std. & \\
\hline Soft splint & 2.47 & 0.5 & 1.28 & 0.4 & 1.09 & 0.1 & $\mathrm{P}<0.001$ \\
\hline Hard splint & 2.39 & 0.6 & 1.21 & 0.5 & 1.06 & 0.24 & $\mathrm{P}<0.001$ \\
\hline 3D digital splint & 2.48 & 0.5 & 1.14 & 0.33 & 1.00 & 0.17 & $\mathrm{P}<0.001$ \\
\hline P value & \multicolumn{3}{|c|}{0.1} & \multicolumn{3}{c|}{$\mathrm{P}<0.001$} & \multicolumn{2}{c}{$\mathrm{P}<0.001$} & \\
\hline
\end{tabular}




\section{DISCUSSIONS}

\section{Discussion of Methodology}

Bruxism is believed to be one of the most common parafunctional habits nowadays, that has been suggested to be an initiating factor for TMD, and myofacial pain disorders ${ }^{(15)}$.

In this study patients suffering from bruxism of myogenic origin were selected, they were suffering from one or more from the following signs and symptoms to help in confirming the myogenic origin: pain in the auricular area, tenderness of masseter and / or temporalis muscle, morning headaches, and pain in the temporomandibular joint region associated with eating ${ }^{(16)}$.

Patients in this study had trigger points, which are localized spots of in which tenderness is felt upon manual pressure. Sustained pressure in this area cause tingling or numbness ${ }^{(17)}$.

Smokers were also excluded as tobacco effects peripheral circulation, and soft tissue healing, impairs the muscle protein synthesis, and increases the central dopaminergic activity leading to increase in the bruxism activity ${ }^{(18-21)}$.

Patients having loss of vertical dimension of occlusion were excluded, as this effects the position of the condyle, leading to excessive load on the TMJ, causing pathological conditions in it, and prevent the jaw muscles from proper functioning due to the change in the distance between the muscles origin and insertion sites especially the masseter and the medial pterygoids muscles ${ }^{(21,22)}$.

Patients age ranges between $18-60$, as very old patients were excluded to avoid atrophy in the skeletal muscles due to senility ${ }^{(23)}$.

The patients were randomly divided into three equal groups, in each group a different treatment modality was used: conventional soft occlusal splint and hard splint and 3Dprinted splint.

- Visual analogue scale VAS was used to evaluate the efficiency of each treatment modality ${ }^{(24,25)}$.
- Objective evaluation: Pain report analysis Muscular palpation (masseter, temporalis and pterygoid muscles) was performed bilaterally with tight and constant pressure and were classified on a scale from 0 to $3 .{ }^{(26)}$

Soft splints have been used as an interim appliance until acrylic-resin splints could be provided. These appliances have also been suggested as prognostic tool to evaluate whether an acrylic-resin splint would be advantageous (27). It has been suggested that the soft occlusal surface of soft splint may contribute to occlusal changes ${ }^{(28)}$. Soft splints have been advocated for patients with temperomandibular disorders (TMD). However, there are few trials that have evaluated efficacy, and outcomes have been variable. Research on TMD recommended the evaluation of pain in the masticatory muscle through subjective pain and digital palpation. ${ }^{(29)}$

Hard splint, is a stabilization splint that has a flat surface that ensures occlusal stability of both dental arches. it is used as it cause relaxation in the masticatory muscle, reposition the disc and the condyle in a physiological position ${ }^{(30)}$.

The flat surface of the splint provides freedom in centric, and allow for realization of self positioning of the mandible $\mathrm{e}^{(30)}$.

A Face bow record was done to help relate the arc of closure e.g. hinge axis of the mandible to the cranium. Then the face bow record was transferred to a semi adjustable articulator helps the articulator to simulate the jaw movements more accurately when the casts where vertically separated ${ }^{(31)}$

The thickness of the splint was $2 \mathrm{~mm}$ thickness, as it has been postulated that a stabilization appliance is more effective when fabricated at the vertical dimension where the muscle has minimal surface EMG activity ${ }^{(32)}$

It was found that the masseter and temporalis muscles have their minimal surface EMG activity at vertical opening ranging from 4.5 to $18 \mathrm{~mm}^{\left({ }^{(32)}\right.}$ 
To test this hypothesis, TMD patients were randomized into three groups.

One group received stabilization appliances with increased vertical dimension only by 1 $\mathrm{mm}$, the second group received appliances with increased vertical dimension $4.4 \mathrm{~mm}$ which is half the opening that produce minimal masseter muscle surface EMG activity, and the third group received appliances with increased vertical dimension 8.2 $\mathrm{mm}$ which represents the opening that produced minimal EMG activity. TMD symptoms resolve. It is generally recommended that the appliances should be fabricated at the range from 1 to $4 \mathrm{~mm}$ thick $^{(32)}$.

Protrusive and lateral records were introduced to the virtual articulator soft ware to allow for fabrication of the canine ramp in order to produce disocclusion between the posterior teeth in both lateral and protrusive movements ${ }^{(30)}$.

The splint was fabricated on the maxillary cast to enhance its retention and stability ${ }^{(30)}$.

The buccal cusps of all the mandibular posterior teeth must be in contact with the flat surface of the splint, to enhance the stability of the splint and to avoid any occlusal changes in the lower $\operatorname{arch}^{(33)}$

\section{Discussion of results}

The cognitive awareness theory suggests that the presence of the splint as a foreign object in the mouth will change the oral tactile stimuli, decrease the oral volume and space for the tongue, and make the patients conscious about the position and potentially harmful use of their jaw. Although it seems reasonable to suppose that this increased awareness would influence the patients' learning to alter or reduce their harmful behavior and therefore contribute to the overall zsuccess of the intervention ${ }^{(34)}$

A third hypothesis was postulated that the increase in the vertical dimension of rest results in muscle relaxation. ${ }^{(35)}$
For the patients receiving hard splint the exact hypothesis for the improvement in muscle is still controversial. Some studies suggest that the splint replace the patient's occlusal disharmonies providing the patient with "interference free" occlusion, others suggest that the appliance makes the patients aware of their habit ${ }^{(36)}$.

Each muscle is rich in array of nociceptors, represented in thin mylinated group III afferent and unmylinated group IV fibers which are responsible for muscle contraction when stimulated by specific sensitizing agents. This sensitizing stimuli could be thermal, mechanical, ischemic, or chemical stimuli $^{(37)}$.

The list of chemicals known to sensitize muscle nocireceptors is extensive and includes bradykinins, serotonin, potassium, prostaglandin $\mathrm{E} 2$, and a variety of neuropeptides, such as substance $\mathrm{P}$ and calcitonin generelated peptide (CGRP) ${ }^{(38)}$.

Prolonged muscle spasm leads to diminish in local blood supply and increase in the muscle demands for oxygen which leads to release in pain producing substances. Upregulated nociceptors may be clustered to form "pain trigger points " as in case of myofacial pain syndrome ${ }^{(37)}$.

Statistically significant improvements in the tenderness of the masticatory muscles were found in all groups throughout the follow-up period. However, tenderness of the masticatory muscles showed a significantly greater percentage of improvement in the soft compared to the hard splint group. ${ }^{(39)}$

Change in tenderness to digital palpation has been shown to be a reliable outcome measure in clinical trials on TMD. The results for objective palpation showed statistically significant difference between baseline and 90 days for both hard and soft splints. For hard splint there was significant difference respectively starting from 7,60 and 90 days. (26) Harkins et al found that $93 \%$ of the 42 patients who were given soft splints and had reduction in their 
symptoms and also reported good to excellent results with the subsequent acrylic-resin splint used during the next 3 to 6 months. He also found that $74 \%$ of the patients who wore a soft splint had a reduction in facial myalgia ${ }^{(40)}$. A high degree of patient acceptance has been reported with soft splints. The soft, resilient material may help dissipate some of the heavy loading that occurs during parafuntional activities. In a study done by Nevarro et al who cited that soft splints are ineffective, our study has found that soft splints are effective in reducing the symptoms of myofacial pain ${ }^{(41}$. A study done on nocturnal EMJ comparison of hard and soft splints reported significantly less effect with soft splint ${ }^{(42)}$, it is. Also in accordance with our study, it was found that all patients improved every time regardless of splint design ${ }^{(43)}$. In other study on the pattern of splint usage no advantage of any particular pattern of splint use was found ${ }^{(44)}$. Study of Suvinen T et al have also shown an improvement after splint therapy ${ }^{(45)}$. Occlusal splint therapy decreased the pain and tenderness in the muscles and joints of the patients in the present study. In contrast to Pettengill et al who did not find any difference between hard and soft splints $^{(46)}$. In our study hard splint was more effective compared to soft splint although soft splints also showed significant reduction in pain. The present study supports the use of hard and soft splints in the management of myofacial pain dysfunction syndrome.

It was found in accordance with other studies that minimal chair side time was needed in fitting the 3 Dprinted appliances and reduced time in adjusting the occlusion. The turn around time for an occlusal splint is 3 to 5 working days and the costs are comparable with current manufacture techniques. Most importantly, patients, comment on the comfortable fit and decreased bulk. They have certainly had improved uptake and compliance with the wear of occlusal splints and night guard type of appliances and their clinical efficacy has been significantly improved. ${ }^{(46)}$

\section{CONCLUSION}

All patients improved over time. the results showed that both hard and soft splints are effective but the hard splint is more effective compared to soft splint .

\section{REFERENCES}

1. Carlsson, Gunnar E and Tomas Magnusson: Management of tempromandibular disorder in the general dental practive $.1^{\text {st }}$ edition, 1999.

2. Jeffrey P.Okson: management of tempromandibular disorders and occlusion. $7^{\text {th }}$ edition, 2013.

3. Nabila Adieb and Abdel Fattah Alibiary: Head and Neck, 69-75, 2002.

4. Bilge Gokfen Rohlig, Selin Kipirdi, UgurMeriq, Nalan Qapan, and HalukKeskin: Masticatory Muscle Pain and Low-Level Laser Therapy: A Double-Blind and PlaceboControlled Study. Turk J Phys Med Rehab, 57:31-37, 2011.

5. Marini I, Gatto MR and Bonetti GA: Effects of superpulsed low level laser therapy on tempromandibular joint pain, Clin J Pain. 26,7:611-616, 2010.

6. Sangeeta Yadav, Jyoti T. Karani: The Essentials of Occlusal Splint Therapy. International Journal of Prosthetic Dentistry. 2,1,2011.

7. Debora BevilaquaGrossi and Thais Cristina Chaves: Physiotherapeutic treatment for tempromandibular disorders (TMD) Braz J Oral Sci. 3, 10, July/September 2004 .

8. Reda A Abdel-Fattah:Oral Appliances for managing TMJ injuries and disorders.South Florida Institute for PostGraduate Health Education.2009

9. Williamson EH: Temporomandibular dysfunction and repositioning splint therapy. Prog Orthod. 6, 2:206-213, 2005.

10. Littner D, Perlman Emodi A and Vinocuor E: Efficacy of treatment with hard and soft occlusal appliance in TMD. RefuatHapehVehashinayim. 21,3:52-58, 94,Jul 2004.

11. Dupont JS Jr and Brown CE: Occlusal splints from the beginning to the present .Cranio,24:141-145,2006.

12. C. Perez, L. Darmouni, J.F. Carlier: The occlusal splint therapy. International journal of stomatology and occlusal medicine .24:141-145,2009.

13. Debora BevilaquaGrossi and Thais Cristina Chaves: Physiotherapeutic treatment for tempromandibular disorders (TMD) Braz J Oral Sci. 3, 10, July/September 2004 . 
14. Venkatesh Naikmasur, Puneet Bhargava, KruthikaGuttal, Krishna Burde: Soft occlusal splint therapy in the management of myofascial pain dysfunction syndrome: A follow-up study. Indian J Dent Res, 19 ,3, 196-203, 2008

15. Koray, O., B. Küçük, and B. Edebeoglu, Etiology of temporomandibular disorder pain. J Orofac Pain, 2009. 21(3): p. 89-94.

16. Yap, A.U., et al., Depression and somatization in patients with temporomandibular disorders. The Journal of prosthetic dentistry, 2002. 88(5): p. 479-484.

17. Hubbard, D.R. and G.M. Berkoff, Myofascial trigger points show spontaneous needle EMG activity. Spine, 1993. 18(13): p. 1803-1807.

18. Shetty, S., et al., Bruxism: a literature review. The Journal of Indian Prosthodontic Society, 2010. 10: p. 141-148.

19. Rohlİg, B.G., et al., Masticatory Muscle Pain and LowLevel Laser Therapy: A Double-Blind and PlaceboControlled Study. Turkish Journal of Physical Medicine \& Rehabilitation/Turkiye Fiziksel Tip ve Rehabilitasyon Dergisi, 2011. 57(1).

20. Jaunarajs, K.L.E., et al., Behavioral and neurochemical effects of chronic L-DOPA treatment on non-motor sequelae in the hemiparkinsonian rat. Behavioural pharmacology, 2010.21(7): p. 627.

21. Okeson, J.P., Management of temporomandibular disorders and occlusion. 2014: Elsevier Health Sciences.

22. Klasser, G.D. and J.P. Okeson, The clinical usefulness of surface electromyography in the diagnosis and treatment of temporomandibular disorders. The Journal of the American Dental Association, 2006. 137(6): p. 763-771.

23. Frontera, W.R., et al., Aging of skeletal muscle: a 12-yr longitudinal study. Journal of applied physiology, 2000. 88(4): p. 1321-1326.

24. Bijur, P.E., W. Silver, and E.J. Gallagher, Reliability of the visual analog scale for measurement of acute pain. Academic emergency medicine, 2001. 8(12): p. 1153-1157.

25. Chowdhury, R.H., et al., Surface electromyography signal processing and classification techniques. Sensors, 2013. 13(9): p. 12431-12466.

26. Dr.Roseline D. Meshramkar, Dr. Krutika Guttal, Miss. Almeida Lydia Roque, Dr.Ramesh K. Nadiger, Dr. Lekha K., Comparative Evaluation of Clinical Performance of Different Kind Of Occlusal Splint Therapy in the Management of Myofascial Pain IOSR Journal of Dental and Medical Sciences (IOSR-JDMS) Volume 14, Issue 8 Ver. IX (Aug. 2015), PP 54-57
27. Wright E.F. An easily fabricated occlusal splint. J Am Dent Assoc. 1988; 117(6):757-8.

28. Singh BP, Berry DC. Occlusal changes following use of soft occlusal splints. J Prosthet Dent 1985; 54:711-5

29. Harkins S, Marteney JL, Cueva O, Cueva L. Application of soft occlusal splints in patients suffering from clicking tempero mandibular joints. J Cranio mandibular Pract 1988; 6:71-5.

30. Badel, T., et al., Initial treatment of prosthetic patients with a Michigan splint. Acta Stomatologica Croatica, 2003. 37(2): p. 207-210.

31. Vinayagam, K., Evaluation Of Arc Of Closure Using Arbitary Ear Piece Face Bow And Semi-Adjustable Articulator An Invivo Study. International journal of Nursing Didactics, 2015. 5(05): p. 07-11.

32. Edward F.Wright, D., MS, MAGD, Manual of Temporomandibular disorders. 1st ed. 2005: Blackwell Munksgaard. 119-171.

33. Okeson, J.P., Management of temporomandibular disorders and occlusion. 2014: Elsevier Health Sciences.

34. Türp, J., F. Komine, and A. Hugger, Efficacy of stabilization splints for the management of patients with masticatory muscle pain: a qualitative systematic review. Clinical oral investigations, 2004. 8(4): p. 179-195.

35. Riyadh Akeel, B., EMG and pain severity evaluation in patients with TMD using two different occlusal devices. The International journal of prosthodontics, 2001. 14(1): p. 1

36. JP., O., Managment of temporomandibular disorders and occlusion, 5th edition. 2003. 112: p. 507-36.

37. Arezzo, J.C., Possible mechanisms for the effects of botulinum toxin on pain. The Clinical journal of pain, 2002. 18(6): p.S125-S132.

38. Mense, S., Nervous outflow from skeletal muscle following chemical noxious stimulation. The Journal of Physiology, 1977. 267(1): p. 75.

39. Sameh A Seifeldin, Khaled A. Elhayes, Soft versus hard occlusal splint therapy in the management of temporomandibular disorders (TMDs).The Saudi Dental Journal,2015.

40. Harkins S, Marteney JL, Cueva O, Cueva L. Application of soft occlusal splints in patients suffering from clicking tempero mandibular joints. J Cranio mandibular Pract 1988; 6:71-5.

41. Nevarro E, Barghi N, Rey R. Clinical evaluation of maxillary hard and resilient occlusal splints. J Dent Res $1985 ; 1246: 313$. 
42. Manns, Miralles, Guerrero: Changes in electrical activity of the postural muscles of the mandible upon varying the vertical dimension. J. Prost het. Dent. 45:438, Apr 1981.

43. Ramfjord, Ash: Occlusion $2^{\text {nd }}$ ed, Philadelphia, W.B. Saunders Co., 1971.

44. Carlson CR, Bertrand PM, Ehrlich AD, Maxwell AW, Burton RG. Physical self-regulation training for the management of temporomandibular disorders. J Orofac Pain 2001; 15:47-55.

45. Suvinen T, Reade P. Prognostic features of value in the management of temporo mandibular joint pain-dysfunction syndrome by occlusal splint therapy. J Prosthet Dent 1989; 61:355-61.

46. Pettengill CA, Growney MR, Schoff R, Kenworthy CR. A pilot study comparing the efficacy of hard and soft stabilizing appliances in treating patients with temporomandibular disorders. J Prosthet Dent 1998; 79:165-8.

47. David B. Dunn, BDS, FRACDS, FPFAMichael B. Lewis, BDS, DClinDent, MRACDS., CAD/CAM Occlusalsplints: A new paradigm.Australasian Dental Practice March/April 2011. 